\title{
Interference Impact on Throughput Performance of Bluetooth Scatternets Under Different Traffic Loads
}

\author{
Khaled Morsi *, Gao Qiang, Xiong Huagang \\ Electronic and Information Engineering School \\ Beihang University, Beijing, 100191, China \\ *Corresponding author: kh_morsi_1974@yahoo.com
}

\begin{abstract}
Multiple Bluetooth piconets are likely to co-exist in a physical environment, supporting many wireless applications. An independently operating Bluetooth piconet will inevitably encounter mutual interference from collocated piconets, which results in individual piconet and overall scatternet performance degradation. In this paper, the throughput performance of Bluetooth piconet is studied and evaluated as the number of interfering piconets is increased considering that the frequency hopping mechanism in Bluetooth piconets is not a totally random process. Several different simulations were performed with varying inter-piconet time offset in order to represent the effects of different packet sizes, symmetric / asymmetric transmission, synchronized / unsynchronized data packets, and different traffic loads with non equal probabilities of collision for different packet sizes. Results proved that certain packets sizes can achieve higher throughput depending on type, size, and number of interferers. Throughput improvement may be achieved in the presence of interference by changing the packet size in the piconet. It was also verified that an optimum traffic load could be reached to achieve maximum throughput for each number of piconets.
\end{abstract}

Keywords- Bluetooth; Scatternets; Interference; Throughput;

\section{INTRODUCTION}

Bluetooth is a low-power, low-cost, open-standard, wireless communication system for implementing PANs. Its technology depends on using short-range radio links to construct a small ad hoc network among communicating nodes called a piconet (formed by one master and up to seven active slaves). By interconnecting existing Bluetooth piconets, larger-scale networks called scatternets are established with nominal speeds of up to $1 \mathrm{Mbits} / \mathrm{s}$. The mutual interference between multiple Bluetooth piconets forming a scatternet was discussed in many previous studies. In a previous work [1], the author investigates the interference between Bluetooth piconets and derives an upper bound on packet error rate. The analysis in [1] assumed all packets to be 1-slot and each piconet is fully loaded. The work in [2] extends the result in [1] by considering the physical location relationship between piconets. In the analytical results proposed in [3], all packet types can coexist in the network, without being necessarily fully loaded. In [4], the analytical results in [3] are improved by taking into account the $\mathrm{FH}$ guard time effect. Through [5], [6], [7] the authors used the idea of probabilistic graphs to give a generalized model of interference for all packet types in both heterogeneous and homogenous cluster of piconets. The algorithms provided by the authors in [8] can optimally choose the packet lengths in accordance with the channel conditions for different frequencies to maximize throughput. The estimation offered in [9] depends on reducing collision rate, hence improving throughput, by combining time slot synchronization with inter-piconet clock offset rather than time slot synchronization only.

The mentioned studies provide an analytical approach to the mutual interference problem in Bluetooth scatternets by considering the frequency hopping process in a Bluetooth piconet is totally random. The already published works also emphasize the fact that a large number of coexisting piconets severely degrades individual piconet or network performance in terms of higher packet error rates and lower aggregate throughput regardless the effect of inter-piconet clock offset and the non equal collision rates for different packet sizes. This paper extends the work in [9] and aims to quantify the potential gains that could be obtained by considering the following:

- The Bluetooth hopping sequence is a pseudo random process that leads to lower collision rate and better distribution of frequency channels over available band.

- Collision rate and throughput are functions in interpiconet clock offset, hence, there is often a different collision rate for each inter-piconet clock offset.

- Different packet sizes have non equal probabilities of collision depending on bit error rate BER of each frequency channel assigned for every time slot.

- Different packet sizes have different interference influence, thus it is important to study the effect of type and size of a packet as an interferer.

- It is possible to reach an optimum traffic load to achieve maximum throughput for each number of piconets since all collocated piconets may not carry identical traffic loads.

The remainder of this paper is organized as follows. The Bluetooth standard is summarized in Section II. A theoretical background for synchronized / unsynchronized piconets, symmetric / asymmetric transmission, and throughput are all illustrated in Section III. In Section IV, simulation results are presented and explained. Final conclusions are in Section V.

\section{THE BLUETOOTH STANDARD}

The Bluetooth standard uses a slow frequency hopping spread spectrum scheme of 79 non-overlapping frequency channels (23 in some countries) with $1 \mathrm{MHz}$ channel spacing in 
the unlicensed $2.4 \mathrm{GHz}$ ISM (Industrial, Scientific, and Medical) band [10]. The standard also defines three basic hop patterns, each of which uses all 79 channels before repeating themselves. Each Bluetooth device has a 28 bit $3.2 \mathrm{kHz}$ freerunning counter (clock). Members of a Bluetooth network, or piconet, hop together among the 79 frequencies (numbered 078) with a sequence that is a function of the master's freerunning clock and the first 28 bits of the master's 48 bit address [10].The hop sequence cycle covers about 23.3 hours, spanning about $64 \mathrm{MHz}$ of spectrum, spreading transmissions over $80 \%$ of the available $79 \mathrm{MHz}$ band, and visiting all frequencies with equal probability [9]. The FH channel is based on Time Division Duplexing (TDD) using time slots of nominal duration of 625 usec. The master transmits on even slots and slaves respond on odd slots. Each time slot uses a different hop frequency with nominal hopping rate of 1600 hops/s [10]. To increase data rate, and intuitively reduce hopping rate, multislot packets are defined to cover 1, 3, or 5 time slots, named DH1, DH3, or DH5, respectively, as depicted in Table I.

TABLE I. CHARACTERISTICS OF SOME BLUETOOTH PACKETS [10]

\begin{tabular}{|c|c|c|c|c|c|c|c|c|c|c|}
\hline \multirow[t]{2}{*}{$\begin{array}{l}\text { Packet } \\
\text { Type }\end{array}$} & \multirow{2}{*}{$\begin{array}{l}\text { Access } \\
\text { Code } \\
\text { (AC) } \\
\text { (bits) }\end{array}$} & \multirow{2}{*}{$\begin{array}{c}\text { Packet } \\
\text { Header } \\
\text { (PH) } \\
\text { (bits) }\end{array}$} & \multirow[t]{2}{*}{$\begin{array}{l}\text { Total } \\
\text { Payload } \\
\text { (bits) }\end{array}$} & \multirow{2}{*}{$\begin{array}{c}\text { User } \\
\text { Payload } \\
\text { (bits) }\end{array}$} & \multirow{2}{*}{\begin{tabular}{|c} 
Guard \\
Time \\
(GT) \\
(usec)
\end{tabular}} & \multirow[t]{2}{*}{$\begin{array}{l}\text { FEC } \\
\text { Rate }\end{array}$} & \multirow[t]{2}{*}{ CRC } & \multirow[t]{2}{*}{$\begin{array}{c}\text { Symmetric } \\
\text { Max. Rate } \\
\text { (kbit/s) }\end{array}$} & \multicolumn{2}{|c|}{$\begin{array}{c}\text { Asymmetric } \\
\text { Max. Rate } \\
\text { (kbit/s) }\end{array}$} \\
\hline & & & & & & & & & Forward & Reverse \\
\hline DH1 & 72 & 54 & 240 & 216 & 259 & No & Yes & 172.8 & 172.8 & 172.8 \\
\hline DH3 & 72 & 54 & 1496 & 1464 & 253 & No & Yes & 390.4 & 585.6 & 86.4 \\
\hline DH5 & 72 & 54 & 2744 & 2712 & 255 & No & Yes & 433.9 & 723.2 & 57.6 \\
\hline
\end{tabular}

As shown in Fig.1, a multi-slot packet will continue using same hop channel as being decided by first time slot. After finishing the multi-slot packet transmission, the system tunes back to the carrier frequency sequence as if only a single slot packet had been transmitted. Using this way, the hop frequency selection is not affected by different packet lengths and unaware slaves, or slaves that miss transmission from/to master, all can still hop synchronously [10].

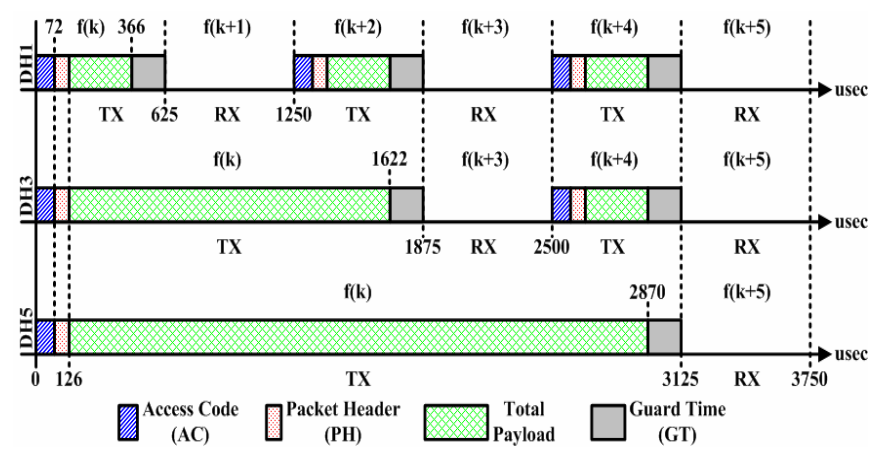

Figure 1. FH/TDD channel characteristics of multi-slot packets

\section{PICONETS SYNCHRONIZATION, TRANSMISSION, AND THROUGHPUT}

To study interference caused by multiple Bluetooth piconet transmissions, throughout this paper, piconet 1 will be subjected to interference from other piconets that are introduced to its environment as interferers. Interference, however, is not guaranteed by just the interferer's presence.
Interference can only occur when piconet 1 and at least one interfering piconet hop to the same frequency during the same time slot. The actual amount of interference will be measured by examining decrease in piconet 1 performance.

\section{A. Piconets Synchronization:}

In this paper, the term "synchronized" will refer to piconets that are aligned in such a way that their time slots are matched up within a few 10's of microseconds. As shown in Fig.2, both synchronized piconets will hop to same frequency channel (78) during third time slot (Packet 3). These two packets will collide with each other, resulting in a retransmission of both packets.

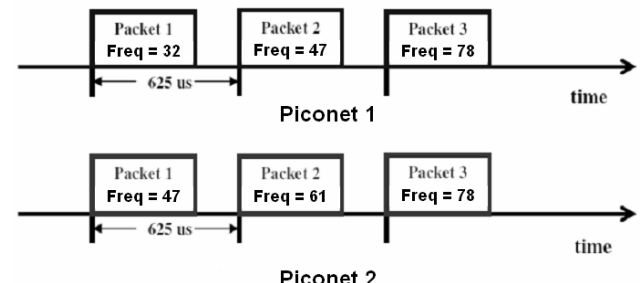

Figure 2. Piconets interference with "synchronized" time slots

If piconets instead have "unsynchronized" time slots, the interference potential will double. As shown in Fig.3, due to misalignment between piconets, and in addition to collision between packet 3 from both piconets on channel 78 as in Fig.2, "packet 2" from piconet 1 and "packet 1" from interfering piconet 2 are now both on same frequency 47 at same time. Hence, in unsynchronized cases, each packet from interfering piconet is a "double threat" to interfere with piconet 1 .

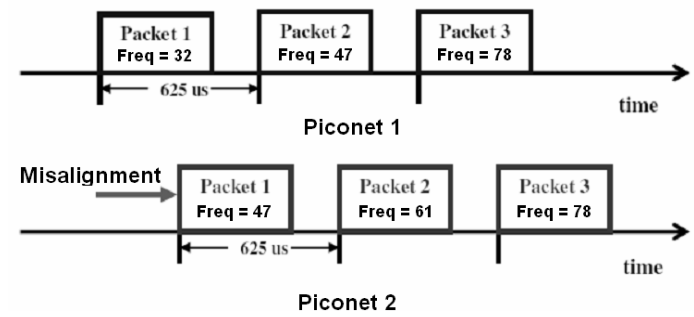

Figure 3. Piconets interference with "unsynchronized" time slots

\section{B. Piconets Transmission:}

The two main classes of Bluetooth transmission links are known as Symmetric and Asymmetric. A symmetric link occurs when both master and slave in a piconet exchange the same sized packet as shown in Fig.4.

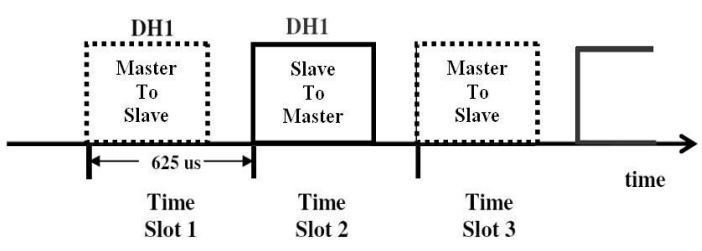

Figure 4. Graphical example of "Symmetric" transmission

An asymmetric link occurs when the master sends one size packet and receives a different size packet from the slave. For 
example, as shown in Fig.5, a 1-slot packet is transmitted from master with slave returning 5-slot packet.

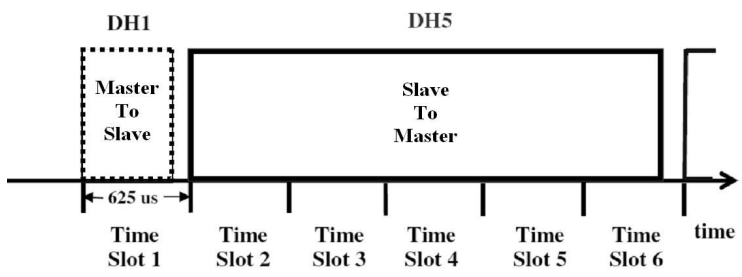

Figure 5. Graphical example of "Asymmetric" transmission

\section{Piconets Throughput:}

For DH1 packets to be successfully received, the transmitted packet and the acknowledgment packet must not suffer from a collision. In fact, the throughput is the probability that one in every pair of consecutive packets is in error. The throughput probability for multiple synchronized piconets is:

$$
\text { Pthroughput }_{\text {sync }}=\left(1-P_{1}\right)^{2 n}
$$

Where $\mathrm{P} 1=(1 / 79)$ is probability of hopping to any of the 79 Bluetooth channels, and $\mathrm{n}$ is number of interfering piconets [1]. For unsynchronized piconets, the possibility for interference will increase, thus, (1) has to be modified. The throughput probability for multiple unsynchronized piconets is:

$$
\text { Pthroughput } t_{\text {unsync }}=\left(1-P_{1}\right)^{4 n}
$$

Where $\mathrm{P} 1=(1 / 79)$ is probability of hopping to any of the 79 Bluetooth channels, and $\mathrm{n}$ is number of interfering piconets [1].

\section{SIMULATION RESULTS}

Simulations have been carried out using MATLAB. The MATLAB program used to generate a Bluetooth hopping sequence given any Bluetooth address and clock start time, is modified to MATLAB FUNCTION "FHSK(clk,add)". The "address" and "clock" inputs correspond to address and clock of master. In order to develop a sequence of frequency hops, the FUNCTION "FHSK(clk,add)" must be repeatedly called as clock is incremented. Since each time slot is a 2 clock cycles long, therefore for example, when transmitting 1-slot packets, the hopping frequency will change every 2 clock cycles.

\section{A. Effect of Traffic Loads}

In order to simulate throughput degradation with increasing number of interfering piconets, data was collected by taking 1000 samples each of 5.12 seconds $\left(2^{13}=8192\right.$ time slots $)$ with increased inter-piconet time offset between samples and equal probabilities of collision for different packet sizes. The result represents mean collision rate, considering random interpiconet time offset. The aggregate throughput was calculated by multiplying throughput per piconet by both total number of piconets and maximum symmetric (2-way) data rates for $\mathrm{DH} 1$, DH3, and DH5 transmissions for both cases, synchronized and unsynchronized piconets. Equations (1) and (2) were used for calculating theoretical aggregate throughputs. All simulated and theoretical results were compared as shown in Fig.6.

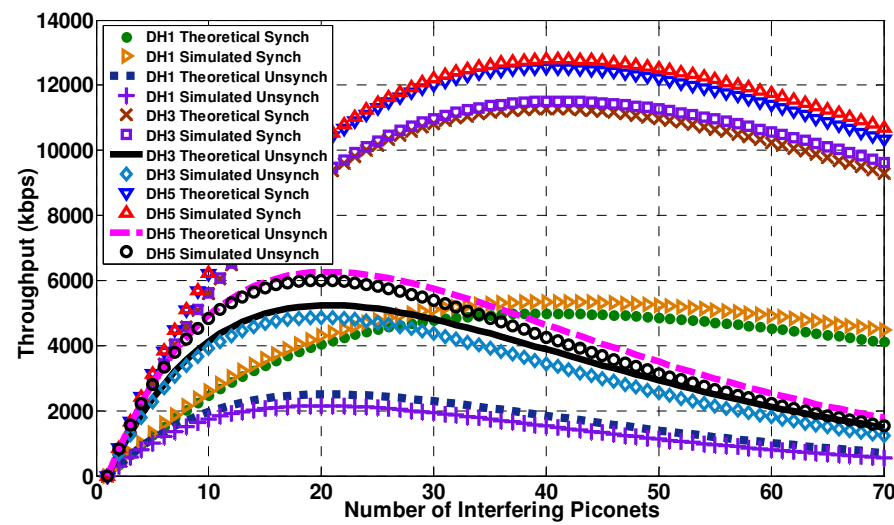

Figure 6. Aggregate throughput versus number of interfering piconets

From Fig.6, it is clear that mean throughput calculated by varying the inter-piconet time offset is equal to theoretical estimations. The curves also show the improvement that can be achieved by synchronizing time slots among piconets. For both synchronized and unsynchronized interferers, the maximum aggregate throughput is achieved with DH5 packets at 40 and 20 piconets, respectively. The maximum throughput doubles from the unsynchronized case to the synchronized case.

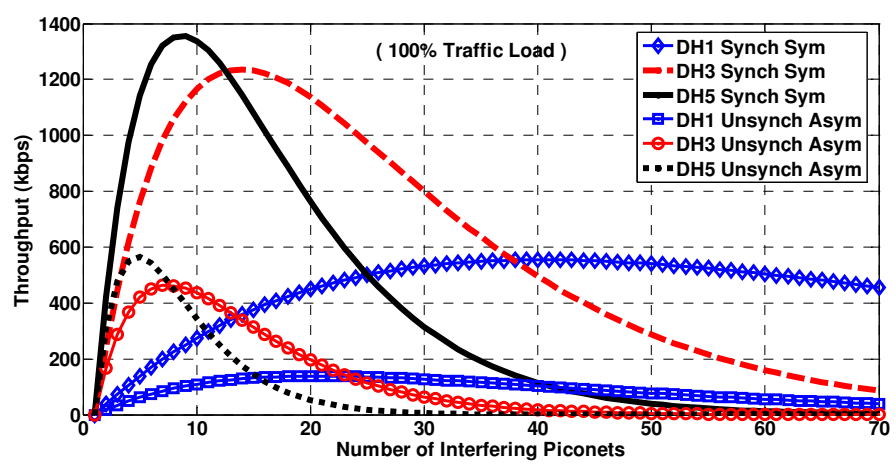

Figure 7. Throughput vs. number of interfering piconets (100\% traffic load)

In order to study the influence of traffic load on mutual interference among collocated piconets, the simulation program was modified and repeated again considering 10, 20, 40, 60, $80,100 \%$ traffic loads. Different packet sizes are considered to have non equal probabilities of collision and inter-piconet time offset is increased between the collected 1000 samples. Results are shown in Fig.7 and Fig.8 for 100\% and 60\% traffic loads.

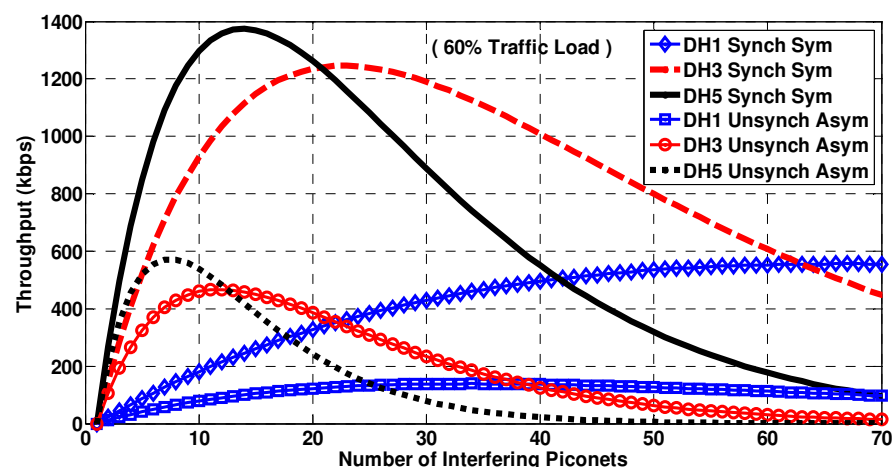

Figure 8. Throughput vs. number of interfering piconets (60\% traffic load) 
The throughput curves shown in Fig.7 and Fig.8 saturate at a certain point as number of piconets increases, and then drops different from earlier observation in Fig.6. Curves also indicate that longer packets are more preferable in terms of throughput because collision problem can be compensated by the benefit of higher bandwidth efficiency. However, at a certain number of piconets, throughputs saturate due to more serious collisions, hence shorter sized packets will be preferred. For example, as shown in table II, for synchronized symmetric transmissions with $60 \%$ traffic load, DH5 packets achieve highest throughput up to 21 piconets, after this point and up to 63 piconets, DH3 packets outperforms DH5 ones. Finally, DH1 packets will be the best choice after 63 piconets.

\section{TABLE II. COMPARISON BETWEEN 100\% AND 60\% TRAFFIC LOADS}

\begin{tabular}{|c|c|c|c|c|}
\hline Transmission & Traffic & \multicolumn{3}{|c|}{ Packet Size for Best Throughput } \\
\cline { 3 - 5 } Type & Load & $\mathrm{DH} 5$ & $\mathrm{DH} 3$ & $\mathrm{DH} 1$ \\
\hline Synchronized & $100 \%$ & $\mathrm{~N}<13$ & $13<\mathrm{N}<38$ & $\mathrm{~N}>38$ \\
\cline { 2 - 5 } \begin{tabular}{c} 
Symmetric \\
\cline { 2 - 5 }
\end{tabular} & $60 \%$ & $\mathrm{~N}<21$ & $21<\mathrm{N}<63$ & $\mathrm{~N}>63$ \\
\hline \hline $\begin{array}{c}\text { Unsynchronized } \\
\text { Asymmetric }\end{array}$ & $100 \%$ & $\mathrm{~N}<8$ & $8<\mathrm{N}<23$ & $\mathrm{~N}>23$ \\
\cline { 2 - 5 } & $60 \%$ & $\mathrm{~N}<13$ & $13<\mathrm{N}<38$ & $\mathrm{~N}>38$ \\
\hline
\end{tabular}

Fig. 9 plots throughput of each traffic load against number of co-existing piconets. It indicates that it is possible to reach an optimum traffic load to achieve maximum throughput for each number of piconets. In other words, this result can be used to estimate the proper number of piconets to be deployed in a physical area, in terms of throughput and traffic load. For example, form Fig.9, if 20 piconets were to form a scatternet, maximum throughput can only be achieved with $60 \%$ traffic load. While with $20 \%$ traffic load, maximum throughput can only be reached at 55 piconets.

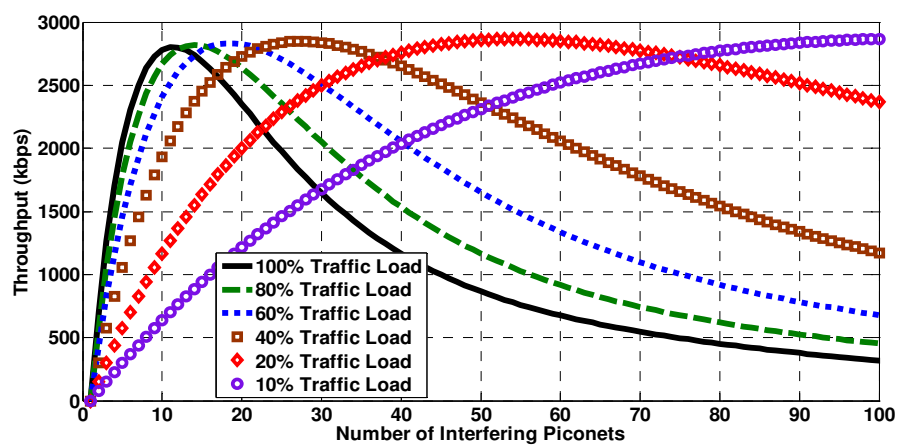

Figure 9. Throughput vs. number of interfering piconets (all traffic load)

\section{B. Effect of Different Types of Interferers}

The simulation program was modified to simulate interference caused by 12 different types of interference accounted for every combination of packet sizes, symmetric / asymmetric transmission and synchronized / unsynchronized time slots. For asymmetric transmission, DH3 or DH5 packets are followed by DH1 response, while DH1 packets were followed by Null response. Once again, data was collected by taking 1000 samples each of 5.12 seconds interval with increased inter-piconet time offset between samples. Fig.10 shows the effects of these different types of interfering transmissions on a Bluetooth piconet transmitting DH1 symmetric data packets.

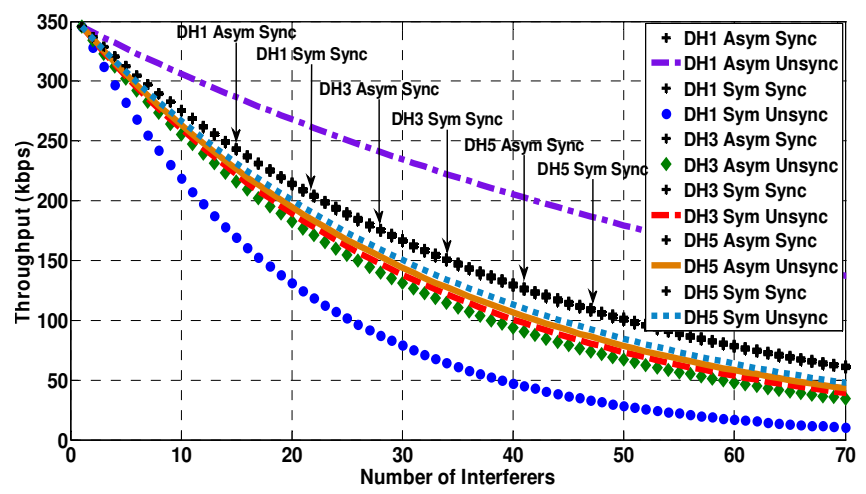

Figure 10. DH1 symmetric packets throughput vs. interfering piconets

From Fig.10, it is clear that least amount of interference occurs when the interfering piconet is transmitting DH1 asymmetric unsynchronized packets. This case represents the most likely and beneficial unsynchronized case where the transmitted packet is a potential interferer. However, the Null response in the interfering piconet is not a threat to interfere at all. This specific type of interference produces the best case throughput curve shown in Fig.10. The next best throughput performance was achieved when the interfering piconets had synchronized time slots. In fact, when time slots between piconets are synchronized, same amount of interference occurs on a DH1 transmission regardless of interferers' packet size. This can be seen on the second curve from top in Fig.10. This curve actually shows the curves from all six synchronized cases overlapping. For unsynchronized piconets, best throughput was achieved when interferers transmit larger packet sizes because double threats will occur less frequently on DH1 packets. A final observation from Fig. 10 is that asymmetric interferers are more threatening than symmetric ones because asymmetric transmissions respond with smaller packets, therefore will have smaller average packet length than symmetric ones. Hence, double threats for interference will occur more frequently.

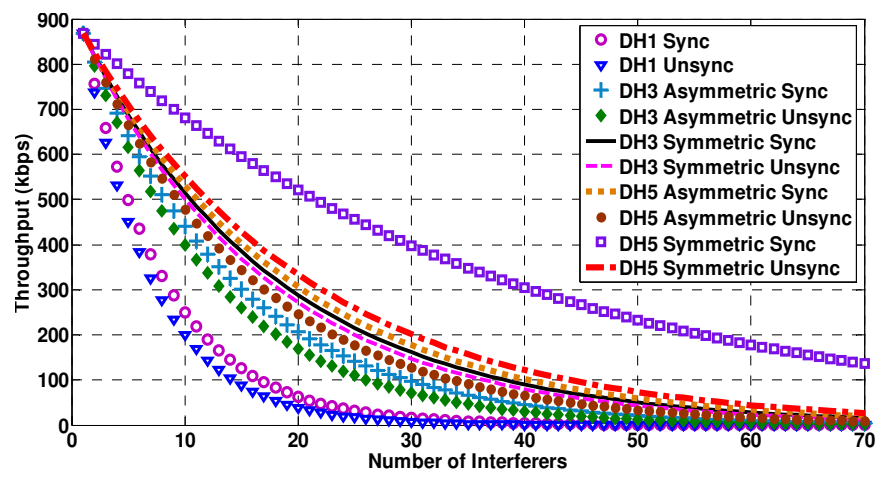

Figure 11. DH5 symmetric packets throughput vs. interfering piconets

The simulation was repeated to test for effects of different interferers types on DH5 symmetric/asymmetric transmissions. Results in Fig.11 and Fig.12 show that DH5 packets were also more susceptible to smaller packet sized interferers. The best throughput was achieved when the interfering piconets used the same type of transmission as "piconet 1". Again, synchronized time slots showed improvement for each interferer type. 


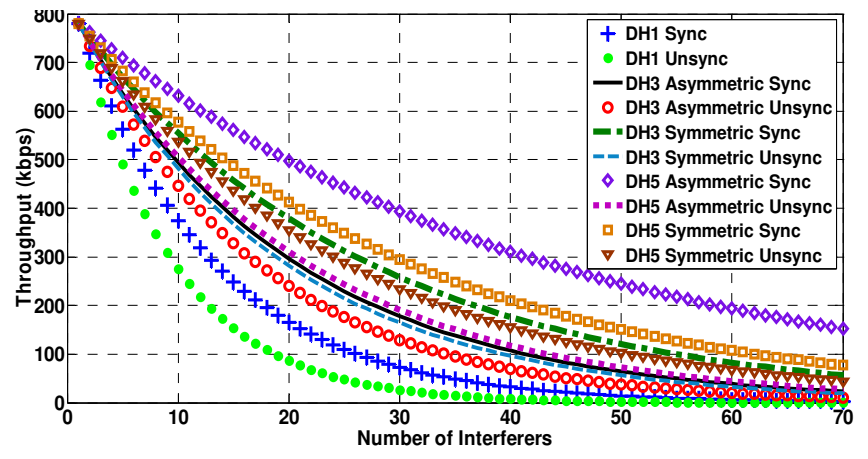

Figure 12. DH5 asymmetric packets throughput vs. interfering piconets

It was shown that certain types of transmissions are more likely to cause inter-piconet interference. Now, it is important to compare types of transmissions to examine which is the most vulnerable to a particular type of interferer. Data used in this analysis were extracted from simulations described previously. Each plot here represents how DH1 and DH5 transmissions hold up against particular types of interferers. Fig.13 shows DH1 interference effect on these packet types.

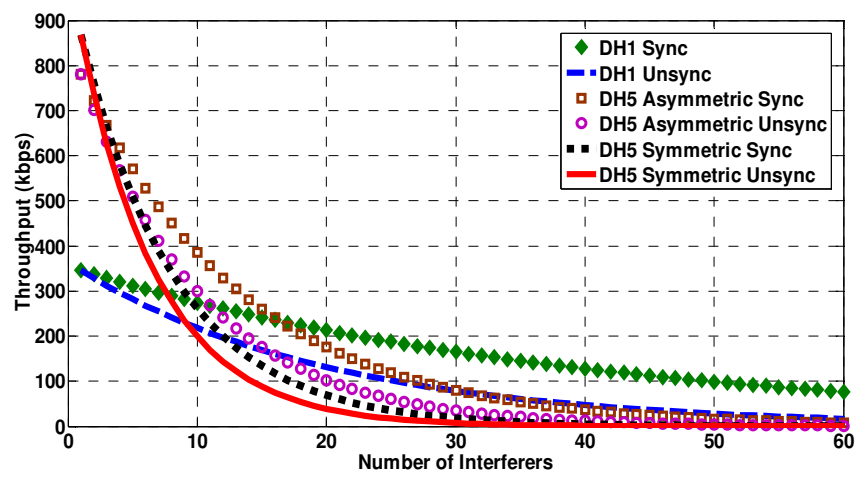

Figure 13. Effect of DH1 interferers on throughput

Curves in Fig.13 show that as number of interfering piconets increase, different types of transmissions can provide higher throughput. Larger data packets are more susceptible to collisions with 1-slot interferers. However, maximum throughput will occur with 5-slot transmissions until there is a significant amount of interference. When there are more than two DH1 interferers present, the total throughput becomes greater through the use of DH5 asymmetric data packets. When number of DH1 interfering piconets surpasses 15, better throughput is achieved by using DH1 transmissions. Fig.13 also verifies the improvement achieved through time slot synchronization. When time slots are synchronized, there is still a point where throughput is larger when transmitting DH5 asymmetric packets. This occurs when there are more than 18 synchronized DH1 interfering piconets present.

Fig.14 shows results of DH5 symmetric interferers. Maximum throughput is achieved using symmetric DH5 transmission up to 10 unsynchronized piconets. After this point, a larger throughput is achieved using DH5 asymmetric transmissions. However, in this case it isn't very advantageous (in terms of 2-way throughput) to use DH1 packets. Again, Fig.14 represents improvement gained by using time slot synchronized; the maximum throughput is always achieved when transmitting DH5 symmetric packets, regardless of the number of interfering piconets. A 2-way throughput of 150 kbps can be achieved with up to 70 interfering piconets present.

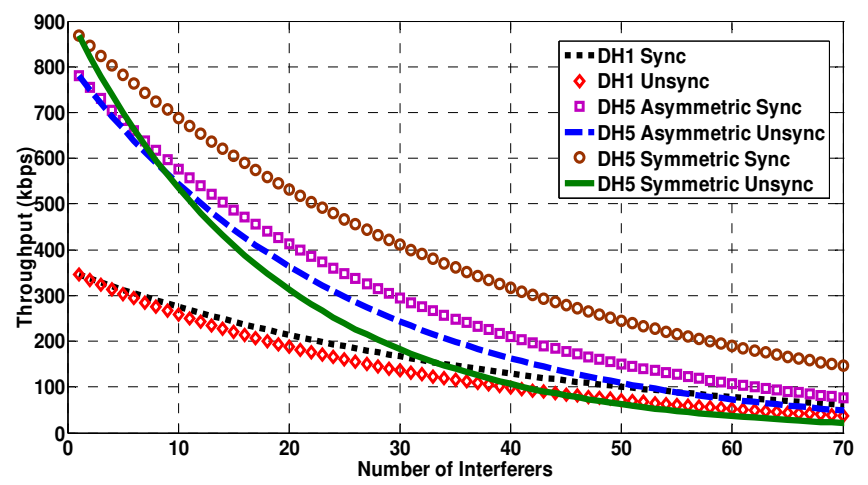

Figure 14. Effect of DH5 symmetric interferers on throughput

\section{CONCLUSIONS}

Single slot packets receive less interference from interfering piconets transmitting larger packets because double threats occur less frequently when interfering piconets are transmitting large packets. Longer packets are more preferable in terms of throughput because collision problem is compensated by the benefit of higher bandwidth efficiency. However, at a certain number of piconets, throughput saturates due to more serious collisions, hence shorter packets will be preferred. An optimum traffic load can be reached to achieve maximum throughput for each number of collocated piconets. Larger packets are more vulnerable to interfering piconets transmitting small packets. Throughput improvement may be achieved in presence of interference by changing packet size in piconet. Certain packets sizes can achieve higher throughput depending on type, size, and number of interferers. However, sometimes throughput is achieved at cost of poor efficiency.

\section{REFERENCES}

[1] A. El-Hoiydi, "Interference between Bluetooth networks-Upper bound on the PER", IEEE Commun. Lett.,5, pp. 245-247,2001.

[2] A. El- Hoiydi and J. D. Decotignie, "Soft deadline bounds for 2-way transactions in Bluetooth piconets under co-channel interference," IEEE Int. Conf. ETFA, Oct. 2001, pp. 144-151

[3] Ting-Yu Lin, Yu-Chee Tseng, "Collision analysis for a multi-Bluetooth pico-cells environment,” IEEE Commun. Lett,7, pp.475-477, Oct. 2003.

[4] Ting-Yu Lin, Yu-Chee Tseng, "An Improved Packet Collision Analysis for Multi-Bluetooth Piconets Considering FH Guard Time Effect," IEEE J. in Commun, 22, pp. 2087-2094, Dec 2004.

[5] K. Naik, D. S. L. Wei, and Y. T. Su, "Packet interference in a heterogeneous cluster of Bluetooth piconets," IEEE vehicular tech. Conf., Oct. 2003, pp. 582-586

[6] K. Naik, D. S. L. Wei, and Y. T. Su, "Analysis of packet interference in a cluster of Bluetooth piconets under different traffic conditions," IEEE Int. Conf. Commun.2004, pp. 3450-3454

[7] K. Naik, D. S. L. Wei, and Y. T. Su, "Analysis of Packet Interference and Aggregated Throughput in a Cluster of Bluetooth Piconets under Different Traffic Conditions," IEEE J. in Commun, V.23, June 2005.

[8] S. Sarkar, F. Anjum, and R. Guka, "Optimal Communication in Bluetooth Piconets," IEEE trans. on vehicular tech, V.54, March 2005

[9] Khaled M., Xiong H., Gao Q., "Performance Estimation and Evaluation of Bluetooth Frequency Hopping Selection Kernel," IEEE int. conf. (ICPCA09), Dec. 2009.

[10] Bluetooth Special Interest Group, "The Bluetooth Specification, Core 2.1+ EDR", July 26, 2007. 